\title{
Attrition Modeling in the Presence of Decoys: An Operations-other-than-War Motivation
}

\author{
D.P. Gaver, P.A. Jacobs \\ Department of Operations Research, Naval Postgraduate School, \\ Monterey, California 93943
}

Received July 1995; revised January 1997; accepted 10 January 1997

\begin{abstract}
Under various operational conditions, in particular in operations other than war (OOTW) or peacekeeping, an intervening force, here Blue, must occasionally engage in attrition warfare with an opposing force, here Red, that is intermingled with noncombatants. Desirably, Red armed actives are targeted, and not the unarmed noncombatants. This article describes some simple Lanchesterian attrition models that reflect a certain capacity of Blue to discriminate noncombatants from armed and active Red opponents. An explicit extension of the Lanchester square law results: Blue's abstinence concerning the indiscriminate shooting of civilians mixed with Red's is essentially reflected in a lower Blue rate of fire and less advantageous exchange rate. The model applies to other situations involving decoys, and reflects the value of a discrimination capability. (C) 1997 John Wiley \& Sons, Inc. Naval Research Logistics 44: 507-514, 1997
\end{abstract}

\section{INTRODUCTION}

Mutual attrition on the battlefield has classically been modeled without accounting for the possible presence of false targets: decoys of low military value intended to divert opponent fire, or even the deliberate dispersal of unarmed civilians among armed and active combatants. The latter is a situation that might well occur in the operations other than war (OOTW) scenarios anticipated as one of the major regional contingencies (MRCs) types into which the U.S. or joint forces could be drawn.

The issue to be addressed herein is that of understanding how Red actives' use of decoys, for example, civilians, for cover, influences Blue's capability to inflict attrition upon Red armed active forces, and at what expense in terms of Blue's own attrition and the inadvertent attrition inflicted, or wasted, upon such decoys. Clearly, attrition of human decoys is to be strenuously avoided for humanitarian reasons, but also because of its broad impact on world opinion; in some circumstances such attrition might well inflame resistance to the extent that the civilian population could itself become an active threat. But in order for Blue to avoid killing Red-controlled civilians, or less politically sensitive targets, that is, to avoid wasting time and resources that could otherwise be directed toward targeting Red actives, some sacrifice in Blue effectiveness must be accepted.

We provide here a preliminary set of simple models for quantifying the effect of substituting discrimination for pure attrition when false targets are present. It will be seen that the 
effect of accounting for target discrimination power by Blue can be reduced to an explicit formula that generalizes the classical Lanchesterian square law. Elaboration to include more realistic detail induces the need for more ambitious numerical work, but the latter is not formidable. Addition of various Blue force and Red decoy types, Blue (inanimate/nonhuman) decoys, stochastics, and the aforementioned change of affiliation by Red (or Blue: slaughter of Red civilians by Blue forces may induce the latter to either slacken their attack, or stimulate greater Red resistance) can all be modeled and ultimately quantified.

\section{INITIAL, AND SIMPLEST, FORMULATION}

Let

$R_{a}(t)=$ number of Red active, attrition-capable, armed military forces at time $t$;

$R_{c}(t)=$ number of Red unarmed civilians or other decoys mixed with the above at $t$; $B(t)=$ number of Blue active armed forces at $t$.

In what follows it is clearly necessary to require that $B(t), R_{a}(t)$, and $R_{c}(t)$ all be nonnegative; otherwise nonsense results occur. The attrition equations are thus nonlinear.

Mechanism of combat: Red actives deplete the Blue actives according to the Lanchester so-called aimed-fire (square-law) equation

$$
\frac{d B}{d t}=-\rho_{R B}(t) R_{a}(t)
$$

The Blue actives attempt to do the same, but must avoid killing civilians, or generally being diverted by decoys.

\subsection{Blue Shoots at First Available Red}

If a Blue simply picks a target Red at random then, assuming military and civilians are well mixed and appear to the Blues in proportion to their numbers, Blue targets an active Red with success probability $s_{a}(t)=R_{a}(t) /\left[R_{a}(t)+R_{c}(t)\right]$, so

$$
\frac{d R_{a}}{d t}=-\rho_{B R}(t) s_{a}(t) B(t)=-\rho_{B R}(t)\left[\frac{R_{a}(t)}{R_{a}(t)+R_{c}(t)}\right] B(t) .
$$

Note that it is to Blue's immediate selfish advantage to discriminate between Red actives and civilians, for in the above $R_{a}(t) /\left[R_{a}(t)+R_{c}(t)\right]$ can well be considerably less than unity, in which case Blue only slowly reduces those shooting at him/her. Red civilians are targeted with probability $1-s_{a}(t)=s_{c}(t)$; the results may be quite unacceptable from Blue's viewpoint, and certainly from that of Red. This is a low-resolution model: differentiation between Blue force types, and coordination of fire capabilities, are important in practice but not addressed here. The payoff is a rather explicit analytical result. 


\subsection{Blue Possesses Discriminatory Powers}

Suppose Blue discriminates between Red actives and Red civilians: $i_{a a}$ is the probability that Blue can identify a Red active if he acquires one; $i_{a c}$ is the probability that he misidentifies it as a decoy (civilian); $i_{c a}$, the probability that a decoy (civilian) is mistaken for an active, and $i_{c c}$, the probability of correct identification of a decoy (civilian), are defined correspondingly. We hope that $i_{a a}$ and $i_{c c}$ are near unity, but there may be a substantial cost in time for this capability. Now $R_{a}(t) i_{a a}$ is the (approximate) number of Red actives correctly identified at $t$, whereas $R_{a}(t) i_{a c}$ is the number incorrectly classed as civilians and not shot at, and $R_{c}(t) i_{c a}$ is the number of civilians targeted through misclassification error. We suppose that each Red active or civilian requires a unit of service per unit of time and the service is processor sharing. Then $R_{a}(t) i_{a a} /\left[R_{a}(t) i_{a a}+R_{a}(t) i_{a c}+R_{c}(t) i_{c a}+R_{c}(t) i_{c c}\right]$ is the fraction of time slice $d t$ spent correctly shooting at Red actives, so

$$
\begin{aligned}
\frac{d R_{a}(t)}{d t} & =-\rho_{B R}(t) \frac{R_{a}(t) i_{a a}(t)}{R_{a}(t)\left(i_{a a}(t)+i_{a c}(t)\right)+R_{c}(t)\left(i_{c a}(t)+i_{c c}(t)\right)} B(t) \\
& =-\rho_{B R}(t) \frac{R_{a}(t) i_{a a}(t) B(t)}{R_{a}(t)+R_{c}(t)}
\end{aligned}
$$

The above holds because $i_{a a}+i_{a c}=1, i_{c a}+i_{c c}=1$. Notice that the identification probabilities $i_{c c}$, et cetera could be made time dependent to represent changes in visibility throughout the conflict. We can also write the attrition equation for decoys, or civilians:

$$
\frac{d R_{c}(t)}{d t}=-\rho_{B R}(t) \frac{R_{c}(t) i_{c a}(t)}{R_{a}(t)+R_{c}(t)} B(t) .
$$

The above formulations assume that the Red actives and civilians are well mixed and hence equally likely to be found by a Blue active; however, once found, a candidate target can be assessed for relevance, but with error. For now we slough off the time-consuming aspects of this process. A model with more states can handle this aspect; see Section 3.

Comment. A possible alternative formulation, pointed out by R.L. Helmbold, treats classification in a Bayesian fashion, that is, by treating $R_{a}(t) /\left[R_{a}(t)+R_{c}(t)\right]$ effectively as Blue's prior for a Red active; hence the denominators in the fractions in (3) and (4) are replaced by $R_{a}(t) i_{a a}+R_{c}(t) i_{c a}$. From our modeling perspective, this model would neglect the time spent by Blue actually scanning and misclassifying the fraction $i_{a c}$ of actives, and correctly classifying the fraction $i_{c c}$ civilian decoys. In effect, Blue effort is viewed in (3) as in part processor shared over all Red units. If we choose to neglect this time-expenditure penalty we can indeed alter our equations: the steps leading to ( 8 ) follow as shown, while later expressions are modified.

To move toward actual solutions, divide (3) by (4):

$$
\frac{\frac{d R_{a}}{d t}}{\frac{d R_{c}}{d t}}=\frac{-\rho_{B R}(t) \frac{R_{a}(t) i_{a a}(t)}{R_{a}(t)+R_{c}(t)} B(t)}{-\rho_{B R}(t) \frac{R_{c}(t) i_{c a}(t)}{R_{a}(t)+R_{c}(t)} B(t)}=\frac{R_{a}(t) i_{a a}(t)}{R_{c}(t) i_{c a}(t)}
$$


So upon division

$$
\frac{\left(d R_{a} / d t\right)}{R_{a}(t)} / \frac{\left(d R_{c} / d t\right)}{R_{c}(t)}=i_{a a}(t) / i_{c a}(t)
$$

For simplicity drop the $i$ time dependency; easy explicit integration gives

$$
\ln R_{a}(t)-\ln R_{a}(0)=\left(\frac{i_{a a}}{i_{c a}}\right)\left(\ln R_{c}(t)-\ln R_{c}(0)\right) .
$$

From this,

$$
R_{c}(t)=R_{c}(0) \cdot\left(R_{a}(t) / R_{a}(0)\right)^{i_{c a} i_{a a}} .
$$

Now plug this into Eq. (3):

$$
\frac{d R_{a}(t)}{d t}=-\rho_{B R}(t) \frac{R_{a}(t) i_{a a} B(t)}{R_{a}(t)+R_{c}(0)\left(R_{a}(t) / R_{a}(0)\right)^{i{ }^{\prime}{ }^{\prime}{ }_{a a}}} .
$$

Equations (1) and (9) constitute a pair of nonlinear first-order differential equations that can be routinely solved numerically, subject to initial and boundary conditions: $0 \leq R_{a}(t)$, $B(t) \leq B(0)$. Closed-form analytical solutions are practically inaccessible, but further information can be found. Divide (9) by (1): we come up with an equation that relates $B(t)$ and $R_{a}(t)$ that can be integrated explicitly. We see that a generalized square law appears.

Proceed to solve for $R_{a}$ in terms of $B$ :

$$
\frac{d R_{a} / d t}{d B / d t}=\frac{-\rho_{B R}(t) \frac{R_{a}(t) i_{a a} B(t)}{R_{a}(t)+\left(R_{c}(0) / R_{a}(0)^{i_{c a}{ }^{\prime} i_{a a}}\right)\left(R_{a}(t)\right)^{i_{c a} / i_{a a}}}}{-\rho_{R B}(t) R_{a}(t)}
$$

We rearrange and get

$$
\left(R_{a}(t)+\left(R_{c}(0) /\left(R_{a}(0)\right)^{i_{c a} / i_{a a}}\right)\left(R_{a}(t)\right)^{i_{c a} / i_{a a}}\right) d R_{a}(t)=\frac{\rho_{B R}(t)}{\rho_{R B}(t)} i_{a a} B(t) d B(t) .
$$

Assume that $\rho_{B R}(t) / \rho_{R B}(t)$ is independent of $t$ and integrate to get, finally,

$$
\begin{aligned}
& \frac{R_{a}^{2}(t)}{2}-\frac{R_{a}^{2}(0)}{2}+\left(\frac{R_{c}(0)}{\left(R_{a}(0)\right)^{i_{c a} / i_{a a}}}\right)\left[\frac{R_{a}(t)^{\left(i_{c a} / i_{a a}\right)+1}}{\left(i_{c a} / i_{a a}\right)+1}-\frac{R_{a}(0)^{\left(i_{c a} / i_{a a}\right)+1}}{\left(i_{c a} / i_{a a}\right)+1}\right] \\
& =\frac{\rho_{B R}}{\rho_{R B}} i_{a a}\left[\frac{B^{2}(t)}{2}-\frac{B^{2}(0)}{2}\right] .
\end{aligned}
$$


This is a generalized square law, valid when positive boundary conditions are respected. Notice that if $R_{c}(0)=0$ we are back to the original square law, immortalized in song and story; see, for instance, [3]. If $R_{c}(0)>0$ but $i_{c a}=i_{a a}$ we also have a new and somewhat different square law. Anderson [1] has also considered the influence of decoys or false targets in a more elaborate setting. His formulation differs from ours in that discrimination capability is not represented by functions $i_{a a}, i_{c a}$ (and their respective complements).

ILLUSTRATION 1: $i_{c a}=i_{a a}, R_{c}(0)=R_{a}(0)$.

This is a pessimistic case for Blue, who has no discriminatory power. But the result is simple:

$$
\frac{R_{a}^{2}(t)}{2}-\frac{R_{a}^{2}(0)}{2}+\left[\frac{R_{a}^{2}(t)}{2}-\frac{R_{a}^{2}(0)}{2}\right]=\frac{\rho_{B R}}{\rho_{R B}} i_{a a}\left[\frac{B^{2}(t)}{2}-\frac{B^{2}(0)}{2}\right]
$$

or

$$
R_{a}^{2}(0)-R_{a}^{2}(t)=\left(\frac{\rho_{B R}}{\rho_{R B}}\right)\left(\frac{i_{a a}}{2}\right)\left[B^{2}(0)-B^{2}(t)\right],
$$

which is a new square-law result.

The above is precisely the same equation that would occur if there were no civilians $\left(R_{c}(0)=0\right)$, but with $\rho_{B R}$, the effective attrition rate of Blue versus Red, replacedreduced-to $\rho_{B R} i_{a a} / 2$. In this case the presence of civilians or decoys has diluted the Blue force's effectiveness by $i_{a a} / 2$, that is, by at least a factor of 2 . Furthermore, civilians are still getting targeted and presumably killed, because $i_{c a}=i_{a a}$, which, it is hoped, is unrealistic in practice. This disadvantage must be overcome by sharpening Blue's perception so as to reduce $i_{c a}$ well below $i_{a a}$, which would allow return (nearly) to classical attrition formulas. Otherwise, more Blue forces would be needed to achieve desired results.

ILLUSTRATION 2: $\quad i_{c a}=0$.

This is optimistic for Red civilian noncombatants: they are never targeted. Note that (12) becomes

$$
\frac{R_{a}^{2}(0)}{2}-\frac{R_{a}^{2}(t)}{2}+R_{c}(0)\left[R_{a}(0)-R_{a}(t)\right]=\frac{\rho_{B R}}{\rho_{R B}} i_{a a}\left[\frac{B^{2}(0)}{2}-\frac{B^{2}(t)}{2}\right] .
$$

From this it is apparent that the surviving Red attacker number, $R_{a}(t)$, increases with $R_{c}(0)$, initial decoy supply, as is intuitive: the presence of Red decoys still interferes with Blue's effectiveness, even though none are actually engaged. 
Table 1. Time until attrition reduces force to $85 \%$ of its initial value. 50 red civilians. $\rho_{B R}=\rho_{R B}=0.1$.

\begin{tabular}{|c|c|c|c|c|c|c|c|}
\hline \multirow[b]{2}{*}{$i_{a a}$} & \multicolumn{2}{|c|}{ Initial forces } & \multicolumn{2}{|c|}{$85 \%$ of initial forces } & \multicolumn{3}{|c|}{$\begin{array}{c}\text { Time until } 85 \% \text { of initial force } \\
\text { are attrited }\end{array}$} \\
\hline & Red actives & Blue & Red actives & Blue & Red actives & Blue & Winner \\
\hline 1 & 100 & 100 & 85 & 85 & 2.6 & 1.6 & Red \\
\hline 1 & 100 & 110 & 85 & 93.5 & 2.3 & 1.8 & Red \\
\hline 1 & 100 & 120 & 85 & 102 & 2.1 & 1.9 & Red \\
\hline 1 & 100 & 130 & 85 & 110.5 & 1.9 & 2.2 & Blue \\
\hline 0.8 & 100 & 130 & 85 & 110.5 & 2.4 & 2.1 & Red \\
\hline 0.8 & 100 & 140 & 85 & 119 & 2.2 & 2.3 & Blue \\
\hline
\end{tabular}

\section{Numerical Examples}

Table 1 presents results of numerically solving equations (1), (2), and (4). The MATLAB [4] version 4.0, fourth- and fifth-order Runge-Kutta-Fehlberg numerical integration method was used. Table 1 displays the times until attrition reduces the Red active force to $85 \%$ of its initial value and times until attrition reduces the Blue active force to $85 \%$ of its initial value. In all cases the initial number of Red actives is 100 and the initial number of Red civilians is 50. The attrition parameters $\rho_{R B}=\rho_{B R}=0.1$. Blue always classifies Red civilians correctly; $i_{c c}=1$.

The side that reaches $85 \%$ of its initial forces first is considered the loser. Even if Blue has perfect classification of active Reds, $i_{a a}=1$, Blue needs more than 20 additional forces to be the winner when there are 50 Red civilians in the area. Furthermore, if Blue does not have perfect classification, $i_{a a}=0.8$, then Blue needs more than 30 additional forces to be the winner.

To compensate for likely variability of outcome and uncertainty, Blue force size to guarantee winning will very likely be much larger than in the above table.

\section{ALTERNATIVE SEEKER-ATTRITER CONFLICT FORMULATIONS}

A more realistic, but also more complex problem formulation explicitly distinguishes between Blue seekers and Blue actives: the former locate Red units, while the latter attack/ attrit those Reds detected and then identified (possibly incorrectly) as actives. We define

$$
\begin{aligned}
R_{a f}(t) & =\text { number of Red actives that are free (undetected by Blue) at time } t \\
R_{a d}(t) & =\text { number of Red actives that are detected by Blue at time } t ; \\
R_{c f}(t) & =\text { number of Red civilians (or decoys) free at time } t ; \\
R_{c d}(t) & =\text { number of Red civilians (or decoys) detected (erroneously) at } t ; \\
B_{s}(t) & =\text { number of Blue seekers at time } t ; \\
B_{a}(t) & =\text { number of Blue actives at time } t .
\end{aligned}
$$

Various issues add to the modeling choices possible; we explore examples only. Specifically, here Blue seekers are modeled as detectors of Reds, but not as followers or trackers: Once a Blue seeker makes contact with a Red it puts it into a detected pool that is available for Blue active attack, but the Blue seeker becomes quickly free again. A plausible alternative 
is that a Blue seeker identifies and labels and binds to a Red until the latter is attacked or released (contact is lost). We omit consideration of this option for the present.

There follow the state transition equations:

$$
\begin{aligned}
& \frac{d R_{a f}(t)}{d t}=\underbrace{\xi_{B R}(a) R_{a f}(t) B_{s}(t)}_{\begin{array}{c}
\text { free active reds detected } \\
\text { by blue seekers }
\end{array}}+\underbrace{\lambda_{d f}(a) R_{a d}(t)}_{\begin{array}{c}
\text { Detected active reds' } \\
\text { track lost (freed) }
\end{array}} \\
& \frac{d R_{a d}(t)}{d t}=\underbrace{\xi_{B R}(a) R_{a f}(t) B_{s}(t)}_{\text {free active reds detected }}-\underbrace{\lambda_{d f}(a) R_{a d}(t)}_{\begin{array}{c}
\text { detected active } \\
\text { reds freed }
\end{array}}-\underbrace{\rho_{B R} \frac{R_{a d}(t) i_{a a}}{R_{a d}(t)+R_{c d}(t)} B_{a}(t)}_{\begin{array}{c}
\text { detected active } \\
\text { reds attrited }
\end{array}}, \\
& \frac{d R_{c f}(t)}{d t}=\underbrace{-\xi_{B R}(c) R_{c f}(t) B_{s}(t)}_{\begin{array}{c}
\text { civilian/decoy } \\
\text { red detected }
\end{array}}+\underbrace{\lambda_{d f}(c) R_{c d}(t)}_{\begin{array}{c}
\text { civilian/decoy } \\
\text { red track lost (freed) }
\end{array}}, \\
& \frac{d R_{c d}(t)}{d t}=\underbrace{\xi_{B R}(c) R_{c f}(t) B_{s}(t)}_{\begin{array}{c}
\text { civilian/decoy } \\
\text { red detected }
\end{array}}-\underbrace{\lambda_{d f}(c) R_{c d}(t)}_{\begin{array}{c}
\text { civilian/decoy } \\
\text { red track lost }
\end{array}}-\underbrace{\rho_{B R} \frac{R_{c d}(t) i_{c a}}{R_{a d}(t)+R_{c d}(t)} B_{a}(t)}_{\begin{array}{c}
\text { detected civilian/decoy } \\
\text { red attrited (mistakenly) }
\end{array}}, \\
& \frac{d B_{s}(t)}{d t}=\underbrace{-\rho_{R B}(s)\left(R_{a f}(t)+R_{a d}(t)\right)}_{\begin{array}{c}
\text { blue seeker attrited by } \\
\text { red actives }
\end{array}}, \\
& \frac{d B_{a}(t)}{d t}=\underbrace{-\rho_{R B}(a)\left(R_{a f}(t)+R_{a d}(t)\right)}_{\begin{array}{c}
\text { blue active attrited by } \\
\text { red actives }
\end{array}} .
\end{aligned}
$$

In the above $\xi_{B R}(a)$ is the parameter governing the rate at which a Blue seeker discovers a free Red active; $\xi_{B R}(c)$ is the corresponding rate of Blue seeker discovery of Red civilian or decoy.

The condition that all state variables be nonnegative must be respected when solving (15) - (20); numerical solution is the only practical option. The rate parameters are selfexplanatory; if desired any or all of these may be made time dependent.

\section{SUMMARY DISCUSSION}

The simple models proposed above account in an explicit way for a significant combat phenomenon: the influence of false targets or decoys upon a (Blue) combatant's attritional effectiveness. They incorporate discrimination power (probability) parameters $\left(i_{a a}, i_{c c}\right)$ that represent the capacity of Blue to correctly identify Red decoys, or, in another interpretation, the capacity of Red to confuse and divert Blue. It is hoped that our article will stimulate further investigation in this important military area, which is of considerable current interest: it investigates a specific aspect of what is presently called information warfare. 


\subsection{Related Work}

A referee has observed a similarity between this article's models and those previously introduced by Taylor [3], and subsequently discussed by Roberts and Conolly [2]. The similarity is that the latter model also stipulates two force types (analogous to our Red, however, for them both are active and attrition capable), versus one (our Blue). But our emphasis differs in that explicit attention is paid to the uncertainty with which the single force (our Blue) can actually determine which Red force type element is currently a possible target. Discrimination parameters (alternatively, a confusion matrix) are introduced to explicitly represent the degree with which the Blue force can avoid being tricked by Red. This feature might desirably be introduced into the Taylor-Roberts and Conolly models; as they now stand [see (1) of Roberts and Conolly] their formulation takes no explicit account of which Red type Blue is likely to be targeting; on a modern battlefield this is an important current issue. Optimization could be carried out with respect to real-time targeting as well as by choice of initial force size.

\section{ACKNOWLEDGMENTS}

We thank Bruce W. Fowler (U.S. Army Missile Command) and Lowell Bruce Anderson (Institute for Defense Analysis) for useful discussions. Model oversimplifications and deficiencies are entirely the responsibility of the authors.

\section{REFERENCES}

[1] Anderson, L.B., “Attrition Formulas for Deterministic Models of Large-Scale Combat,' Naval Research Logistics, 42, 345-373 (1995).

[2] Roberts, D.M., and Conolly, B.W., "An Extension of the Lanchester Square Law to Inhomogeneous Forces with an Application to Force Allocation Methodology," Journal of the Operational Research Society, 43, 741-752 (1992).

[3] Taylor, J.G., Force-on-Force Attrition Modeling, Military Applications Section, Operations Research Society of America, Arlington, VA, 1980.

[4] The Math Works, Inc., MATLAB Reference Guide, The Math Works, Inc., Natick, MA, August 1992. 